\title{
Effects of chronic dexamethasone administration on hyperglycemia and insulin release in goats
}

Liqiong Niu, Qu Chen, Canfeng Hua, Yali Geng, Liuping Cai, Shiyu Tao, Yingdong Ni and Ruqian Zhao

\begin{abstract}
Background: Dexamethasone (Dex), a synthetic glucocorticoid, is among the most commonly used drugs worldwide in animals and humans as an anti-inflammatory and immunosuppressive agent. GC has profound effects on plasma glucose level and other metabolic conditions. However, the effect of prolonged use of Dex on glucose metabolism in ruminants is still unclear.

Results: Ten goats were randomly assigned to two groups: the control goats were injected with saline, and the Dex-treated goats were intramuscularly injected daily for $21 \mathrm{~d}$ with $0.2 \mathrm{mg} / \mathrm{kg}$ Dex. The results showed that plasma glucose and insulin concentrations were significantly increased after Dex administration $(P<0.05)$. Additionally, the content of hepatic glycogen was also markedly increased in Dex-treated goats $(P<0.01)$, while the content of glycogen in dorsal longissimus was unchanged by $\operatorname{Dex}(P>0.05)$. The expression of several key genes, involved in blood glucose regulation, was detected by real-time PCR in the small intestine, skeletal muscle and liver. The expression of glucose transporter type 2 (GLUT2), sodium-glucose transporter 1 (SGLT1) and sodium-potassium ATPase (Na-K/ATPase) in the small intestine were generally increased by Dex, and GLUT2 mRNA expression was significantly up-regulated $(P<0.05)$. In liver, the expression of genes involved in gluconeogenesis including glucose6-phosphatase catalytic subunit (G6PC), cytosolic form of phosphoenolpyruvate carboxykinase (PCK1) and pyruvate carboxylase $(P C)$, were significantly down-regulated by Dex. However, the protein expression levels of PCK1 \& PCK2 were significantly increased by Dex, suggesting a post-transcriptional regulation. In dorsal longissimus, the mRNA expression of genes associated with gluconeogenesis and the insulin signaling pathway were generally upregulated by Dex, but the mRNA expression of two markers of muscle atrophy, namely F-box protein 32 (FBXO32/ Atrogin 1) and muscle RING-finger protein 1 (MuRF1), was not altered by Dex.
\end{abstract}

Conclusions: Taken together, these results indicate that chronic administration of a low dosage of Dex induces hyperglycemia mainly through gluconeogenesis activation in the goat liver.

Keywords: Blood glucose, Dexamethasone, Gluconeogenesis, Goat, Liver

\section{Background}

The hypothalamic-pituitary-adrenal (HPA) axis plays an important role in the homeostatic maintenance of blood glucose under normal or stress conditions [1]. Glucocorticoids (GCs), the end-hormones of the HPA axis, play a vital role in regulating glucose homeostasis [2]. Dexamethasone (Dex), a synthetic GC drug, is widely used as an anti-inflammatory and anti-allergic agent to treat

\footnotetext{
* Correspondence: niyingdong@njau.edu.cn

Key Laboratory of Animal Physiology \& Biochemistry, Nanjing Agricultural University, Nanjing 210095, People's Republic of China

various inflammatory, allergic and autoimmune diseases $[3,4]$. However, high doses or chronic use of GCs can produce undesired side effects, such as central obesity, hepatic hyperlipidemia, hypertension, glucose intolerance and muscular atrophy $[5,6]$. Accordingly, these adverse effects to the host often limit the utility of chronic GC use.

As in non-ruminant animals, glucose is an important metabolic nutrient metabolism in ruminants, and also a primary precursor in the synthesis of lactose, which determines milk yield [7]. Ruminants normally absorb only small amounts of dietary glucose in the small intestine 
due to the extensive fermentation of carbohydrates in the forestomachs [8]. As a result, ruminants primarily depend on hepatic gluconeogenesis as their source of glucose to meet their energy demand, utilizing precursors like propionate and glucogenic amino acids [9]. Chronic treatment with GC drugs, such as Dex, has been associated with hyperglycemia and insulinemia in both animal and human studies $[10,11]$. These undesirable GCinduced effects have been focused on the liver, skeletal muscle and adipose tissue. The studies show that GCs induce peripheral insulin resistance, in vivo and in vitro [10-12], by increasing hepatic glucose output and decreasing the peripheral glucose uptake. However, the effects of GC-induced insulinemia are still contentious. For instance, after GC exposure, blood insulin concentration has been found to be increased [13], unaltered [14] or decreased in mammals [15]. It has been reported that Dex reduces insulin secretion by pancreatic $\beta$ cells due to the oxidative stress on these cells, and $\beta$ cells have been found to be particularly vulnerable and susceptible to reactive oxygen species (ROS) toxicity [16]. In cows, a single Dex injection induced a sharp increase in blood glucose concentration and then quickly returned to normal level [17]. The milk-reducing effect of Dex in cows is well known [18] and is related to its ability to decrease glucose uptake by skeletal muscle, adipose tissue as well as the mammary gland, which leads to an insulin resistance state [11, 18]. However, to date, data about the effects of chronic exposure to Dex on glycometabolism are scarce in ruminants. Accordingly, this research focused on evaluating glycometabolic effects on an experimental model of chronic exposure to $\mathrm{GC}$ in goats.

\section{Methods}

\section{Animals and experimental procedures}

Ten healthy male goats, $25 \pm 1.0 \mathrm{~kg}$ in body weight, were raised in individual stalls in a conventional animal feeding housing facility at Nanjing Agricultural University (Nanjing, China). Body weight was measured at 1, 7, 14 and $21 \mathrm{~d}$ before the beginning of Dex administration, and food intake was measured daily during the experiment. The animals were fed twice daily at 08:00 and 18:00 $\mathrm{h}$, and had free access to water during the experimental period. All goats received a high-grain diet composed of $43 \%$ corn, $5 \%$ wheat bran, $17 \%$ mixed concentrate and $35 \%$ forage. Animals were adapted to all procedures of sampling and treatment before treatments. The dose of Dex was determined based on a previous study by Emikpe et al. [19]. The ten goats were randomly assigned to two groups: the control (Con) group was intramuscularly injected with saline, and the Dextreated group was intramuscularly injected with Dex $0.2 \mathrm{mg} / \mathrm{kg}$ at $07: 30 \mathrm{~h}$. before morning feeding for $21 \mathrm{~d}$.

\section{Sample collection}

Plasma samples were obtained on d 1, 7, 14 and 21 of the experiment shortly before the injection and morning feeding from the jugular vein. Samples of the duodenum, dorsal longissimus muscle and liver tissue were collected at the end of the experiment. After an overnight fast, all goats were weighed and killed by an injection of xylazine $(0.5 \mathrm{mg} / \mathrm{kg}$ of body weight; Xylosol; Ogris Pharma, Wels, Austria) and pentobarbital $(50 \mathrm{mg} / \mathrm{kg}$ of body weight; Release; WDT, Garbsen, Germany). Immediately after slaughtering, the duodenum, dorsal longissimus muscle and liver tissue samples were collected and frozen immediately in liquid nitrogen and stored at $-80{ }^{\circ} \mathrm{C}$ for subsequent extraction of RNA and proteins.

\section{Measurement of plasma parameters}

Plasma glucose was measured using an automatic biochemical analyzer (7020, Hitachi, Tokyo, Japan), following the manufacturer's instructions. Plasma insulin was determined using a RIA insulin kit (Beijing North Institute of Biological Tec., Beijing, China), following the manufacturer's instructions. The sensitivity of the assay was $1 \mu \mathrm{IU} / \mathrm{mL}$, the intra-assay coefficient of variation $(\mathrm{CV})$ was $6.9 \%$, and all samples were measured in the same assay to avoid interassay variability. Plasma cortisol was determined using a RIA cortisol kit (Beijing North Institute of Biological Tec.), following the manufacturer's instructions. The sensitivity of the assay was $1 \mathrm{ng} / \mathrm{mL}$, the intra-assay $\mathrm{CV}$ was $7.0 \%$, and all samples were measured in the same assay to avoid interassay variability. Glycogen was measured using a Glycogen kit (Jiancheng Co. Ltd., Nanjing, China), following the manufacturer's instructions. Glucagon was determined with a Glucagon ELISA kit (Feiya Co. Ltd., Nanjing, China), following the manufacturer's instructions. The sensitivity of the assay was $1 \mu \mathrm{IU} / \mathrm{mL}$, the intra-assay $\mathrm{CV}$ was $6.5 \%$, and all samples were measured in the same assay to avoid interassay variability.

\section{Staining for PAS}

The isolated dorsal longissimus muscle and liver tissue samples were washed three times with normal saline and fxed with a $10 \%$ formalin solution for $48 \mathrm{~h}$ at $4{ }^{\circ} \mathrm{C}$, dehydrated with ethanol, and embedded with paraffin. The paraffn-embedded slices were deparaffinized, hydrated with distilled water and oxidized for $10 \mathrm{~min}$ at $40^{\circ} \mathrm{C}$ in a $0.5 \%$ periodic acid solution. The sections were then washed with distilled water, immersed in Schiff reagent, washed in distilled water, counterstained with Mayer's hematoxylin, washed in distilled water, dehydrated in a gradient of ethanol and ultimated sealed using a synthetic mounting medium. The slices of each staining were all were viewed under a light microscope (DM3000 LED; Leica, Wetzlar, Germany). 


\section{RNA isolation, CDNA synthesis and real-time PCR}

Total RNA was extracted from each tissue samples using the TRIzol reagent (15596026; Invitrogen, Shanghai, China). The concentration and quality of the RNA were assessed with a NanoDrop ND-1000 Spectrophotometer (Thermo Fisher Scientific, Waltham, MA, USA). Then $2 \mu \mathrm{g}$ of total RNA were treated with RNase-Free DNase (M6101; Promega, Madison, WI, USA) and reverse transcribed according to manufacturer's instructions. Realtime PCR was performed in an Mx3000P qPCR system (Agilent Technologies, Santa Clara, CA, USA) using the Stratagene Mx3000P qPCR kit (Stratagene, La Jolla, CA, USA). The $18 \mathrm{~S}$ RNA, which is not affected by the experimental factors, was chosen as the reference gene. In order to control the PCR efficiency, two microliters of diluted cDNA $(1: 40, v / v)$ was used for real-time PCR. The PCR protocols were as follows: initial denaturation $\left(1 \mathrm{~min}\right.$ at $\left.95{ }^{\circ} \mathrm{C}\right)$, then a three-step amplification stage (20 s at $95{ }^{\circ} \mathrm{C}, 20-30 \mathrm{~s}$ at $62{ }^{\circ} \mathrm{C}, 30 \mathrm{~s}$ at $72{ }^{\circ} \mathrm{C}$ ) was repeated 45 times. All the primers used are listed in Table 1, and were synthesized by the Tsingke Company (Nanjing, China). The qPCR products were all directly sequenced. Additionally, melt curves were checked to confirm the specificity of the primers. The method of 2 $\triangle \triangle \mathrm{Ct}$ was used to analyze the real-time PCR data, and gene mRNA levels were expressed as the fold change relative to the mean value of the control group.

\section{Western blot analysis}

A total $100 \mathrm{mg}$ of frozen liver tissue was minced and homogenized in $1 \mathrm{~mL}$ of ice-cold RIPA buffer containing the protease inhibitor cocktail Complete EDTA-free (Roche, Penzberg, Germany). The homogenates were centrifuged at $12,235 \times g$ for $20 \mathrm{~min}$ at $4{ }^{\circ} \mathrm{C}$ and then the supernatant fraction was collected. After extraction, protein was diluted to the proper concentration with RIPA buffer. The protein concentration was determined using a BCA Protein Assay kit (Pierce, Rockford, IL, USA). Then, $50 \mu \mathrm{g}$ of protein extract from each sample was separated by $10 \%$ SDS-PAGE, and the separated proteins were transferred onto nitrocellulose membranes (BioTrace; Pall Corp., New York, NY, USA). After transfer, membranes were blocked in blocking buffer with $5 \%$ skim milk for $2 \mathrm{~h}$ at room temperature. Next, the membranes were incubated overnight at $4{ }^{\circ} \mathrm{C}$ with the following primary antibodies: rabbit-anti-PCK1 (1:1,000; 16754-1-AP, Proteintech, Rosemont, IL, USA), mouseanti-PCK2 (1:1,000; ab70359, Abcam, Cambridge, UK), and anti-TUBULIN $\alpha$ (1:10,000; BS1699, Bioworld Technology, Saint Louis Park, MN, USA) in dilution buffer, which was consisted of $3 \%$ bovine serum albumin. After several washes in tris-buffered-saline with Tween, the membranes were incubated in dilution buffer for $2 \mathrm{~h}$ at room temperature with goat anti-rabbit HRP-conjugated or anti-mouse HRP-conjugated secondary antibodies (1:10,000; Bioworld Technology). Finally, the blot was washed and the proteins were detected by enhanced chemiluminescence using the LumiGlo substrate (Super Signal West Pico Trial Kit; Pierce) and the signals were recorded by an imaging System (Bio-Rad, Hercules, CA, USA) and analyzed with the Quantity One software (Bio-Rad).

\section{Statistical analysis}

Data are presented as the mean \pm SEM. The data were tested for normal distribution and analyzed by the Student's unpaired t test or analysis of variance (ANOVA) using the SPSS software package (SPSS version 19.0 for Windows; SPSS Inc., Chicago, IL, USA). Data were considered statistically significant when $P<0.05$.

\section{Results}

Dry matter intake (DMI) and body weight were decreased by Dex

As shown in Fig. 1, the dry matter intake (DMI) (Fig. 1a) was significantly decreased after $7 \mathrm{~d}$ of Dex treatment compared with the control goats $(P<0.05)$. Also, the body weight (Fig. 1b) of Dex-treated goats was significantly decreased after $14 \mathrm{~d}$ compared with the control goats $(P<0.05)$.

\section{Plasma glucose and insulin concentrations were increased by Dex}

As shown in Fig. 1, the plasma glucose concentration (Fig. 2a) was significantly increased after $7 \mathrm{~d}$ of Dex treatment compared to the control goats $(P<0.05)$. The Dex treatment at all time points induced a gradual increase of blood glucose from the first injection onwards, and showed a statistical significance from the injection at $2 \mathrm{~h}(P<0.05)$ (Fig. $2 \mathrm{~b})$, and after $21 \mathrm{~d}$ of injections, the blood glucose was higher in the Dex-treated goats than in the control goats at any time point $(P<0.01)$ (Fig. 2c). Consistently, the changes in the plasma insulin concentration by Dex showed the same pattern as those in the plasma glucose (Fig. 3a and b). The plasma glucagon concentration was not affected by Dex (Fig. 3c), but the ratio of insulin to glucagon was significantly increased at $7 \mathrm{~d}$ and $21 \mathrm{~d}$ in the Dex-treated animals compared with the control goats (Fig. 3d).

\section{Plasma cortisol concentrations were decreased by Dex}

As shown in Fig. 3, plasma cortisol concentrations was similar in the two groups before the experiment, but it was significantly decreased $(P<0.05)$ after $21 \mathrm{~d}$ of Dex treatment (Fig. 4), indicating that there is a strong negative feedback control of endogenous cortisol secretion. 
Table 1 PCR primers for glucose metabolism genes

\begin{tabular}{|c|c|c|c|}
\hline Gene & Sequence $5^{\prime} \rightarrow 3^{\prime}$ & GenBank accession no. & Product length, bp \\
\hline \multirow[t]{2}{*}{ GLUT1 } & Forward: AACCGCAACGAGGAGAACC & NM_001314223.1 & 155 \\
\hline & Reverse: TGCAGCACCACGGCAAT & & \\
\hline \multirow[t]{2}{*}{ GLUT2 } & Forward: GAGGCATATCAGGACTCTAC & XM_005675321.3 & 156 \\
\hline & Reverse: AGGGCACCAATAGCAC & & \\
\hline \multirow[t]{2}{*}{ SGLT1 } & Forward: CACCCATCGCAGCAGT & XM_018060890.1 & 199 \\
\hline & Reverse: CGGGCGTCTTGAATGT & & \\
\hline \multirow[t]{2}{*}{ Na-KVATPase } & Forward: CCTCGAAATCCATTGCTTATACC & [38] & 144 \\
\hline & Reverse: GACCATGTCCGTTCCCAAGT & & \\
\hline \multirow[t]{2}{*}{ GLUT4 } & Forward: GGCGGATGCTATGGGTC & JQ343218.1 & 122 \\
\hline & Reverse: ACGGGTTTCAGGCACTTT & & \\
\hline \multirow[t]{2}{*}{ PCK1 } & Forward: ACGCGCTTCCCGAATTCTCA & XM_004014441.1 & 130 \\
\hline & Reverse: TCCCCAACCTCTITAGTGAC & & \\
\hline \multirow[t]{2}{*}{ PCK2 } & Forward: AACAGCAGGGACTCATCCGAAA & XM_015096868.1 & 100 \\
\hline & Reverse: ATCACCGTCTTGCTCTCTACTCGT & & \\
\hline \multirow[t]{2}{*}{$P C$} & Forward: TCGCACCATGTATGTCATCCC & NM_177946 & 187 \\
\hline & Reverse: AGGCTIIITAAAGGCAGAGGG & & \\
\hline \multirow[t]{2}{*}{$H K$} & Forward: GCGGCTCTCTGATAAAACTCTGTTA & AM492192 & 139 \\
\hline & Reverse: TGAGCCATCGGGAATAGACCTTAC & & \\
\hline \multirow[t]{2}{*}{ IGF1R } & Forward: GGCTCAACCCAGGGAA & XM_018065947.1 & 164 \\
\hline & Reverse: CACTATCAACAGAACCGCAAT & & \\
\hline \multirow[t]{2}{*}{$\mathbb{R}$} & Forward: GCCCTGGTGTCACTTTCC & XM_018051134.1 & 182 \\
\hline & Reverse: GCTGCCTTAGGTTCTGGTT & & \\
\hline \multirow[t]{2}{*}{ IRS1 } & Forward: TGCCTGACCAGCAAGACCA & XM_018058864.1 & 187 \\
\hline & Reverse: CACCTGCATCCAGAACTCCC & & \\
\hline \multirow[t]{2}{*}{ PI3K } & Forward: CGAGCATTTCTGCTTTGGG & XM_018047551.1 & 152 \\
\hline & Reverse: GGTCTTGGAGGCATTGTTCTG & & \\
\hline \multirow[t]{2}{*}{ AKT } & Forward: CTAAGCAGCGGCTTGGTG & NM_001285750.1 & 165 \\
\hline & Reverse: GGTCAGGTGGCGTAATGGT & & \\
\hline \multirow[t]{2}{*}{ Atrogin 1} & Forward: TAAACTTGTGCGATGCTAC & XM_005688865.3 & 167 \\
\hline & Reverse:TGTCATGTGCTCGGATT & & \\
\hline \multirow[t]{2}{*}{ MURF1 } & Forward:GAGCAAGGCAGGTGAAGG & XM_018058864.1 & 134 \\
\hline & Reverse: TGGCACGGCAAGATGAC & & \\
\hline \multirow[t]{2}{*}{ G6PC } & Forward:AATGTCATGTTGTGGTTGGGATTCT & EF062861 & 158 \\
\hline & Reverse: GCATTGTAGATGCTCTGGATGTGG & & \\
\hline \multirow[t]{2}{*}{ GAPDH } & Forward:GGGTCATCATCTCTGCACCT & HM043737.1 & 180 \\
\hline & Reverse: GGTCATAAGTCCCTCCACGA & & \\
\hline \multirow[t]{2}{*}{185} & Forward:GTGATGGGGATCGGGGATTG & [39] & 172 \\
\hline & Reverse: GTAGCGACGGGCGGTGTGTA & & \\
\hline
\end{tabular}

GLUT1 Glucose transporter type 1, GLUT2 Glucose transporter type 2, SGLT1 Sodium glucose transporter type 1, Na-K/ATPase Sodium-potassium ATPase, GLUT4 Glucose transporter type 4, PCK1 Cytosolic form of phosphoenolpyruvate carboxykinase, PCK2 Mitochondrial form of phosphoenolpyruvate carboxykinase, PC Pyruvate carboxylase, HK Hexokinase, IGFIR Insulin-like growth factor 1 receptor, IR Insulin receptor, IRS1 Insulin receptor substrate1, PI3K Phosphoinositide 3-kinase, AKT AKT serine/threonine kinase 1, Atrogin 1 F-box protein 32, MuRF1 Muscle RING-Finger protein 1, G6PC Glucose-6-phosphatase, GAPDH

Glyceraldehyde-3-phosphate dehydrogenase 

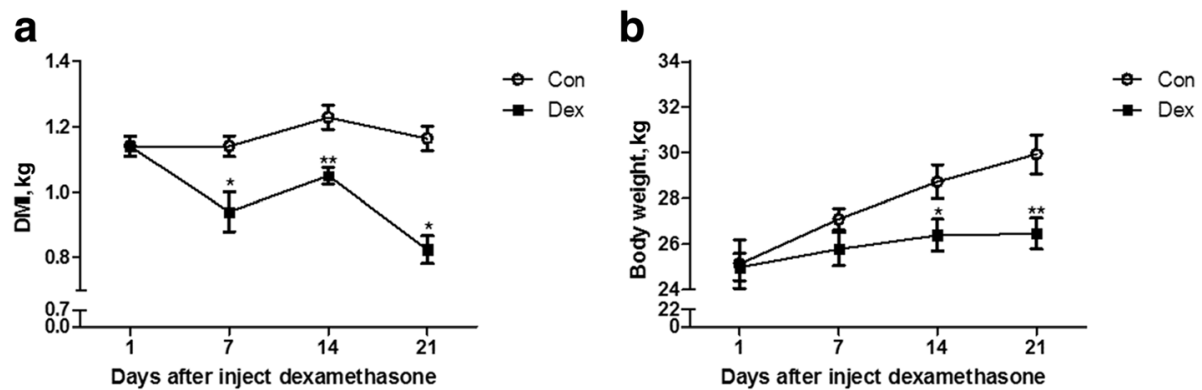

Fig. 1 Changes in body weight and dry matter intake (DMI) in animals treated with dexamethasone (Dex). a, the dynamic changes of DMI during Dex treatment. $\mathbf{b}$, the dynamic changes of body weight during the Dex treatment. Data are presented as the mean \pm SEM. The data were analyzed by the independent-samples $t$-test using the Compare Means of the SPASS 19.0 software for Windows (StaSoft Inc., Tulsa, OK, USA). "** indicates $P<0.05 ; "$ "**" indicates $P<0.01$

\section{The glycogen content in liver and dorsal longissimus} muscle

As shown in Fig. 4a, the content of glycogen in dorsal longissimus muscle was not changed by Dex treatment (Fig. 5a). On the other hand, hepatic glycogen content in Dex-treated goats was significantly increased compared with control goats $(P<0.01)$ (Fig. 5b).

\section{Expression of genes controlling glucose absorption in the duodenum}

The expression of genes encoding proteins controlling glucose absorption in the duodenum was generally upregulated by Dex. Among these genes, GLUT2 mRNA expression was significantly increased by Dex $(P<0.05)$, indicating a higher glucose absorption ability by the small intestine in Dex-treated goats compared with the control counterparts (Fig. 6).

The changes of glucose up-take and insulin signal pathway in dorsal longissimus muscle after Dex administration

The glucose transporter 4, encoded by GLUT4 is the gate for controlling glucose uptake from blood into skeletal muscle. GLUT4 expression was found to be increased in dorsal longissimus muscle in Dex-treated goats, but the increased did not reach statistical significance compared with that in the control (Fig. 7a). Genes associated with the insulin signaling pathway, including
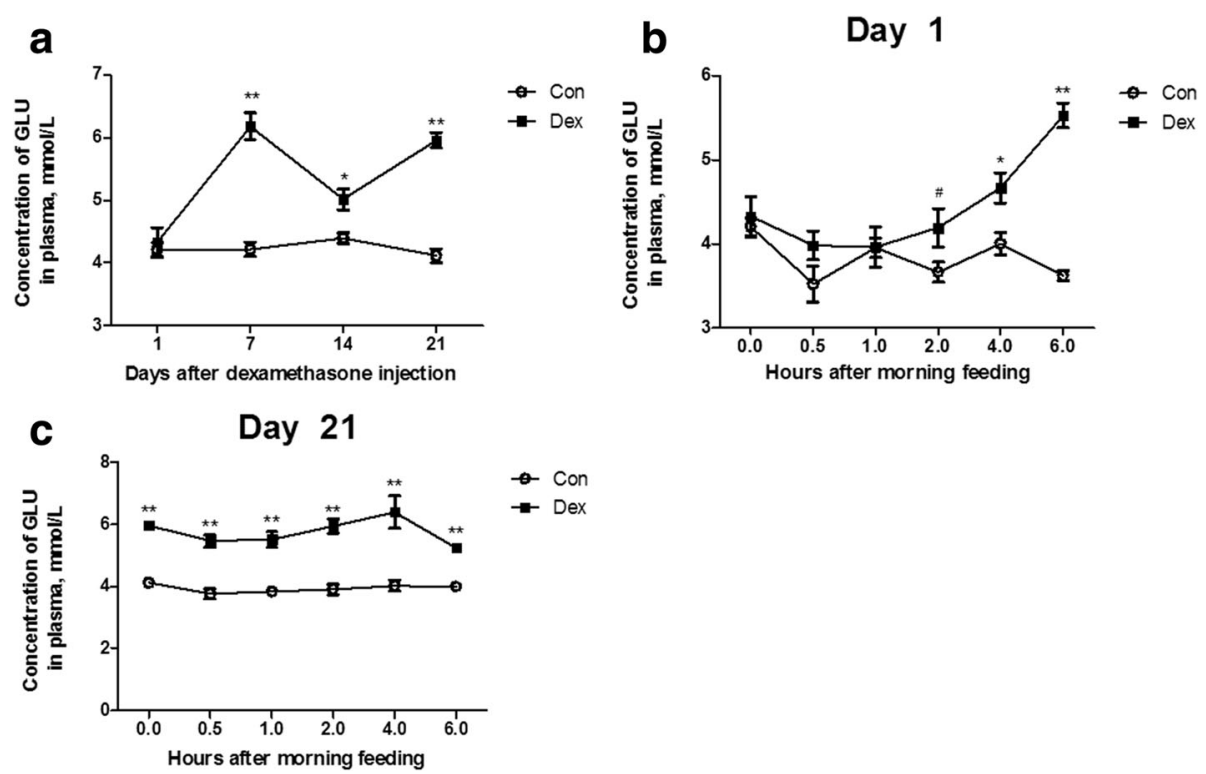

Fig. 2 Chronic dexamethasone (Dex) treatment induced hyperglycemia in goat. a, the dynamic changes of plasma glucose during Dex treatment. $\mathbf{b}$, the dynamic changes of plasma glucose after the first injection of Dex injection. $\mathbf{c}$, the dynamic changes of plasma glucose after 21 $\mathrm{d}$ of Dex treatment. Data are presented as the mean \pm SEM. The data were analyzed by the independent-samples $t$-test using the Compare Means of the SPASS 19.0 software for Windows (StaSoft Inc., Tulsa, OK, USA). "\#" indicates $0.1<P<0.05$; "**" indicates $P<0.05$; "**" indicates $P<0.01$ 

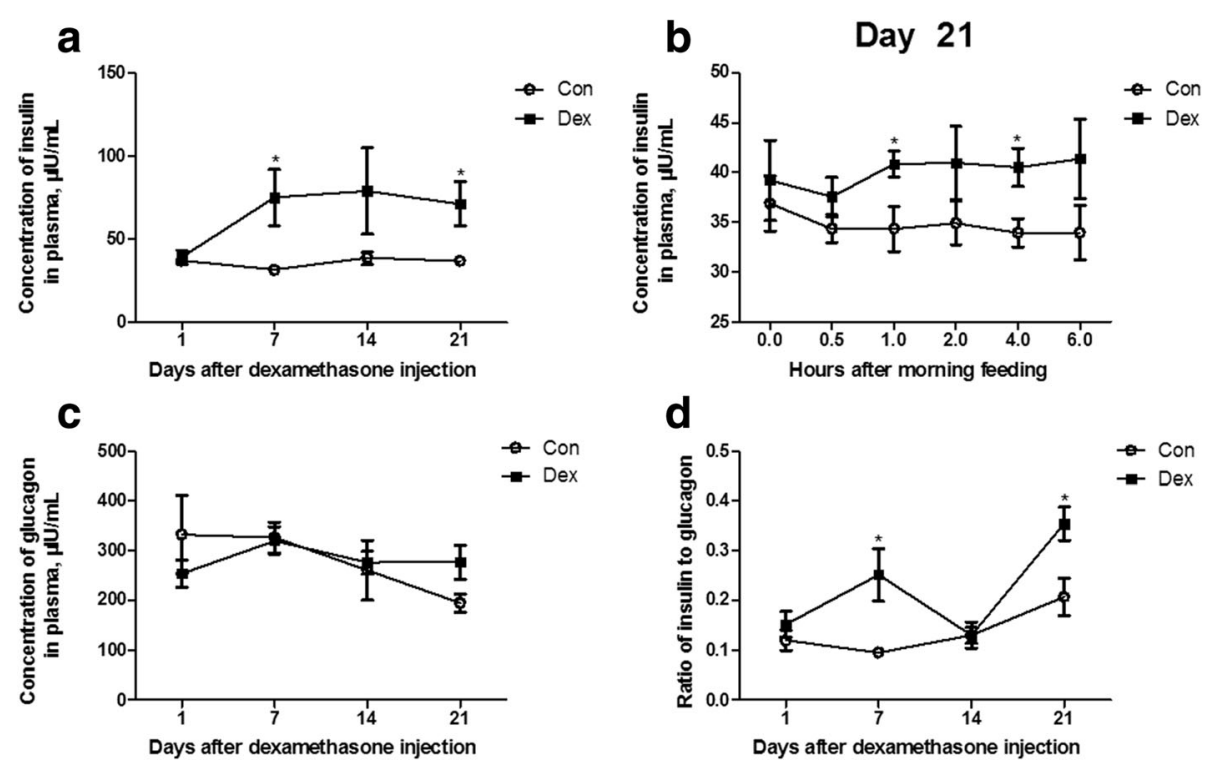

Fig. 3 Chronic dexamethasone (Dex) treatment induced higher level of plasma insulin in goat. a, the dynamic changes of plasma insulin during Dex treatment. $\mathbf{b}$, the dynamic changes of plasma insulin after $21 \mathrm{~d}$ of Dex treatment. $\mathbf{c}$, the dynamic changes of plasma glucagon during Dex treatment. $\mathbf{d}$, the dynamic changes of ratio of insulin to glucagon in plasma during Dex treatment. Data are presented as the mean \pm SEM. The data were analyzed by the independent-samples $t$-test using the Compare Means of the SPASS 19.0 for software Windows (StaSoft Inc., Tulsa, OK, USA). "**" indicates $P<0.05$

the insulin-like growth factor 1 receptor (IGF1R), insulin receptor (IR), insulin receptor substrate1 (IRS1), phosphoinositide 3-kinase (PI3K) and AKT serine/threonine kinase $1(A K T / P K B)$ were also significantly up-regulated by Dex (Fig. 7b). Expression of Atrogin1 and MuRF1, two markers of muscle atrophy, were unaltered by Dex at the transcriptional level (Fig. 7c).

\section{Gluconeogenesis was enhanced in liver by Dex}

Real-time PCR analysis results showed that the expression of several key genes involved in hepatic

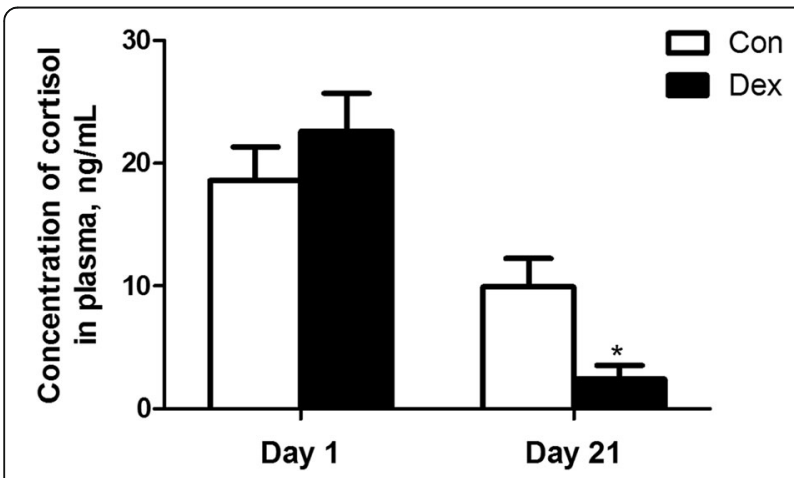

Fig. 4 Chronic dexamethasone (Dex) exposure decreased plasma cortisol concentration in goat. Data are presented as the mean \pm SEM. The data were analyzed by the independent-samples $t$-test using the Compare Means of the SPASS 19.0 software for Windows (StaSoft Inc., Tulsa, OK, USA). "**" indicates $P<0.05$ gluconeogenesis including G6PC, $P C K 1$ and $P C$ mRNA was markedly down-regulated by Dex $(P<0.05)$ (Fig. 8a). However, the protein level of PCK1 and the mitochondrial form of phosphoenolpyruvate carboxykinase (PCK2) protein detected by Western blot analysis were significantly increased in the liver of Dex-treated goats compared with the control $(P<0.05)$ (Fig. $8 b)$, suggesting a post-transcriptional regulation mechanism.

\section{Discussion}

In this study, we found that goats chronically exposed to Dex, a synthetic GC, exhibited a reduction in body weight, hyperglycemia and hepatic glycogen accumulation, which is consistent with similar findings by studies in other mammals. Dex treatment was found to reduce cortisol concentration in all mammals. It is well established that GCs exert feedback inhibition on their own secretion by blocking the effects of the corticotrophin releasing hormone on the pituitary gland $[20,21]$. In this study, a significant decrease of endogenous cortisol secretion was observed in Dex-treated goats. These observations demonstrated that an experimental model of chronic GC administration to goats has been well established, and Dex-treated goats showed similar metabolic conditions as observed in other mammals.

The reduction in body weight was accompanied by a decrease in feeding and an increase in water consumption. The increase in water consumption may indicate a higher energy expenditure. Other studies indicate that 

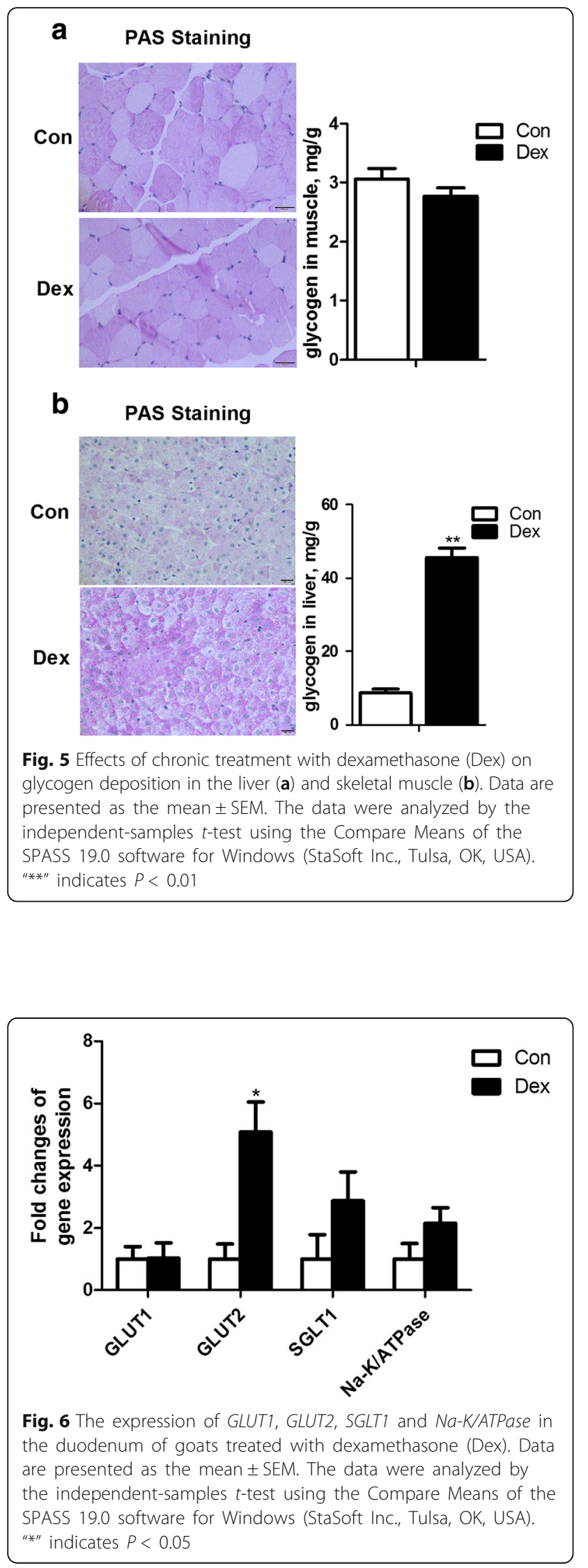

Dex can reduce body weight without affecting food ingestion by increasing caloric expenditure [22]. GCinduced skeletal muscle atrophy through the decrease of the rate of protein synthesis and increase of the rate of protein breakdown has been reported [23]. The GCstimulated atrophy is mediated through the increased expression of several Atrogenes ("genes involved in atrophy"), such as Atrogin1, and MuRF1 [23]. In this study, we did not find a significant difference in expression of these two genes in skeletal muscle of goats after chronic Dex treatment. Thus, the decrease in food intake and higher energy expenditure may lead to the lower body weight of Dex-treated goats. However, the occurrence of GC-induced muscle atrophy still cannot be excluded in this study, and needs further investigation.

The main metabolic effect of GCs is to positively regulate the supply of enough glucose into the circulation to fuel the brain and ensure survival of the organism under conditions of acute stress or starvation. These effects are critical during stress, which in the short run does not affect or even enhances glucose tolerance. However, chronic exposure to GC results in hyperglycemia and insulin resistance [24]. Moreover, other studies have reported that there was a marked increase in the ratio of insulin to glucagon in Dex-treated rats [25]. Our results are consistent with the available research in human and non-ruminant animals. In this study, we observed a transient increase in blood glucose, insulin and the insulin to glucagon ratio, which might be explained by an increase in the hepatic glycogen output resulting from the stimulation of gluconeogenesis and leads to an increase in peripheral resistance to insulin. Glucocorticoids exert tissue-specific effects on glucose metabolism. In the liver, GCs promote hepatic gluconeogenesis [26], however, they reduce glucose uptake and utilization in skeletal muscle and white adipose tissue [27], which coordinately contribute to hyperglycemia and peripheral insulin resistance. In ruminants, glucose is an important nutrient of metabolism [7], however, only a small amount of blood glucose is absorbed in the small intestine. Also, the source of glucose depends on hepatic gluconeogenesis by utilizing some precursors such as propionate produced from fermentation in rumen and glucogenic amino acids to meet their metabolic demand for glucose [9]. The conversion of propionate carbon to glucose is controlled by the abundance of $P C K 2$ and $G 6 P C$ [28]. In non-ruminant mammals, Dex significantly increased gluconeogenic genes of PCK1 and G6PC expression in hepatocytes via binding to the GCs responsive elements (GREs) of the PCK1 and G6PC genes [29]. In this study, however, the expression of hepatic gluconeogenic genes mRNA were significantly decreased by chronic treatment with Dex, this discrepancy is explained by differences between the species. In contrast, 

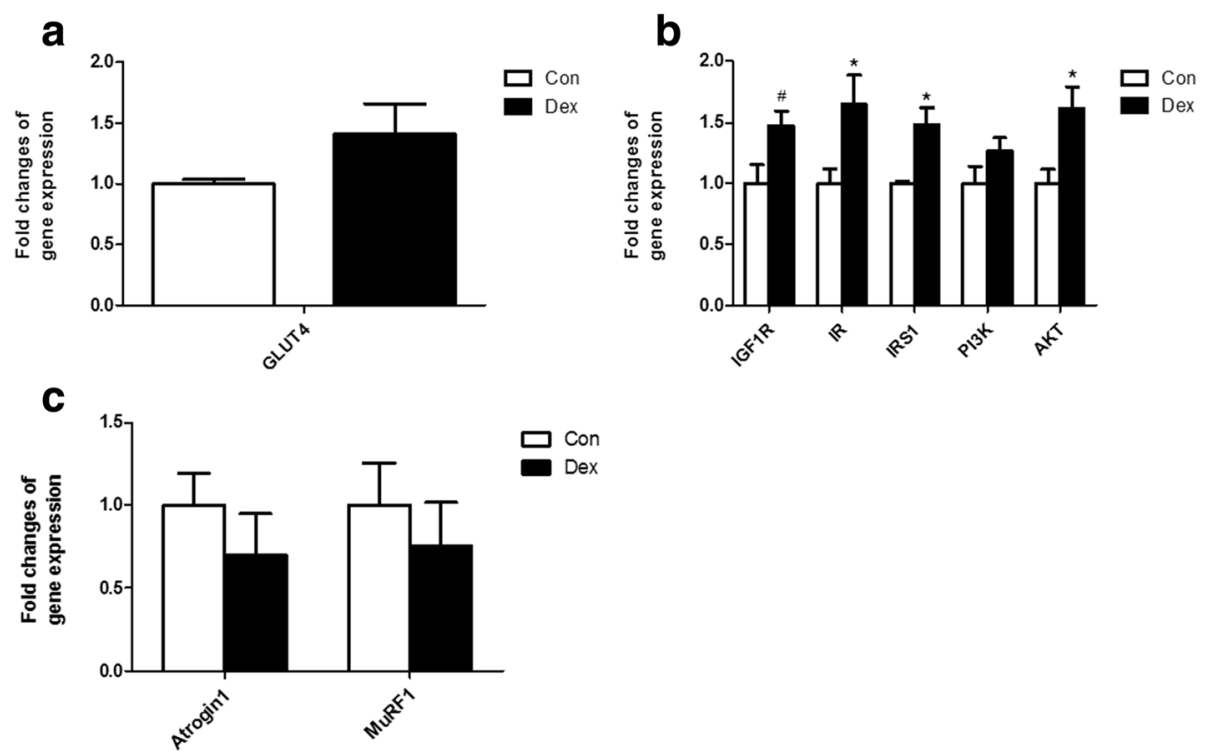

Fig. 7 Relative gene expression in dorsal longissimus muscle. a, gene expression of GLUT4 in skeletal muscle after Dex treatment. b, Expression of IGFTR, IR, IRS1, PI3K and AKT in goat muscle after Dex treatment. c, Expression of Atrogin1 and MURF1 in skeletal muscle after Dex treatment. Data are presented as the mean \pm SEM. The data were analyzed by the independent-samples $t$-test using the Compare Means of the SPASS 19.0 software for Windows (StaSoft Inc., Tulsa, OK, USA). "\#" indicates $0.1<P<0.05$; "**" indicates $P<0.05$

the protein expression level of PCK1 and PCK2 was markedly increased in the liver of Dex-treated goats compared with normal control goats, suggesting the involvement of a post-transcriptional regulation by Dex, as well as a higher ability of hepatic gluconeogenesis in Dex-treated goats. Moreover, our results also showed a significant increase of hepatic glycogen accumulation in goats chronically exposed to Dex, which is consistent with research in other mammals [30,31].

It's well documented that GCs exert tissue-specific effects on glucose metabolism. Glucocorticoids inhibit glucose utilization by reducing both glucose uptake and oxidation in skeletal muscle and white adipose tissue, two major tissues involved in insulinresponsive glucose uptake [32, 33]. In the liver, several studies suggest that GC increases glycogen storage, whereas in skeletal muscle GC plays a permissive role for catecholamine-induced glycogenolysis or inhibit insulin-stimulated glycogen synthesis [34]. In contrast, Burke et al. [35] reported that when mice were chronically exposed to corticosterone, the expression of glycogen synthase 1 was greatly enhanced in muscle which was consistent with elevations in muscle glycogen storage. The present study revealed that Dex moderately increased muscular GLUT4 mRNA expression and overall upregulated the expression of glucogeogenic genes in goat skeletal muscle, however, the content of muscular glycogen content in goats was not found to be changed by Dex. One possible explanation for these findings is that the activities and protein abundances of muscular gluconeogenic enzymes are not coupled with their genes expression, as observed in liver of Dex-treated goats. Also, in this study, the expression of genes involved in the insulin signaling pathway was generally up-regulated by chronic exposure to Dex. Indeed, the results indicated a common insulin resistance induced by Dex in peripheral tissues, which was consistent with the significantly higher level of blood glucose and insulin in Dex-treated goats.

Unlike non-ruminant mammals, ruminants only absorb small amount of glucose in the small intestine. Here, a significant up-regulation of the expression of GLUT2 mRNA was detected in Dex-treated goats. Some studies have reported that GCs increase the intestinal absorption of sugars in both young and mature rats [36]. Additionally, chronic stress was found to significantly increase the expression of GLUT2 in the rat duodenum, which results in the increase of sugar up-take and contributes to the development of hyperglycemia [37]. Accordingly, it is reasonable to assume that the increase of GLUT2 expression may facilitate sugar up-take in the duodenum epithelial membrane in Dex-treated goats, which may partially contribute to causing hyperglycemia. However, the mRNA expression of SGLT1, as well as GLUT1 and Na-K/ATPase was not altered by chronic exposure of goats to Dex. In ruminants, whether the adverse effects of chronic exposure to GC on metabolic disorders can be reversed by steroid removal as observed in rats [35], still waits for further study. 


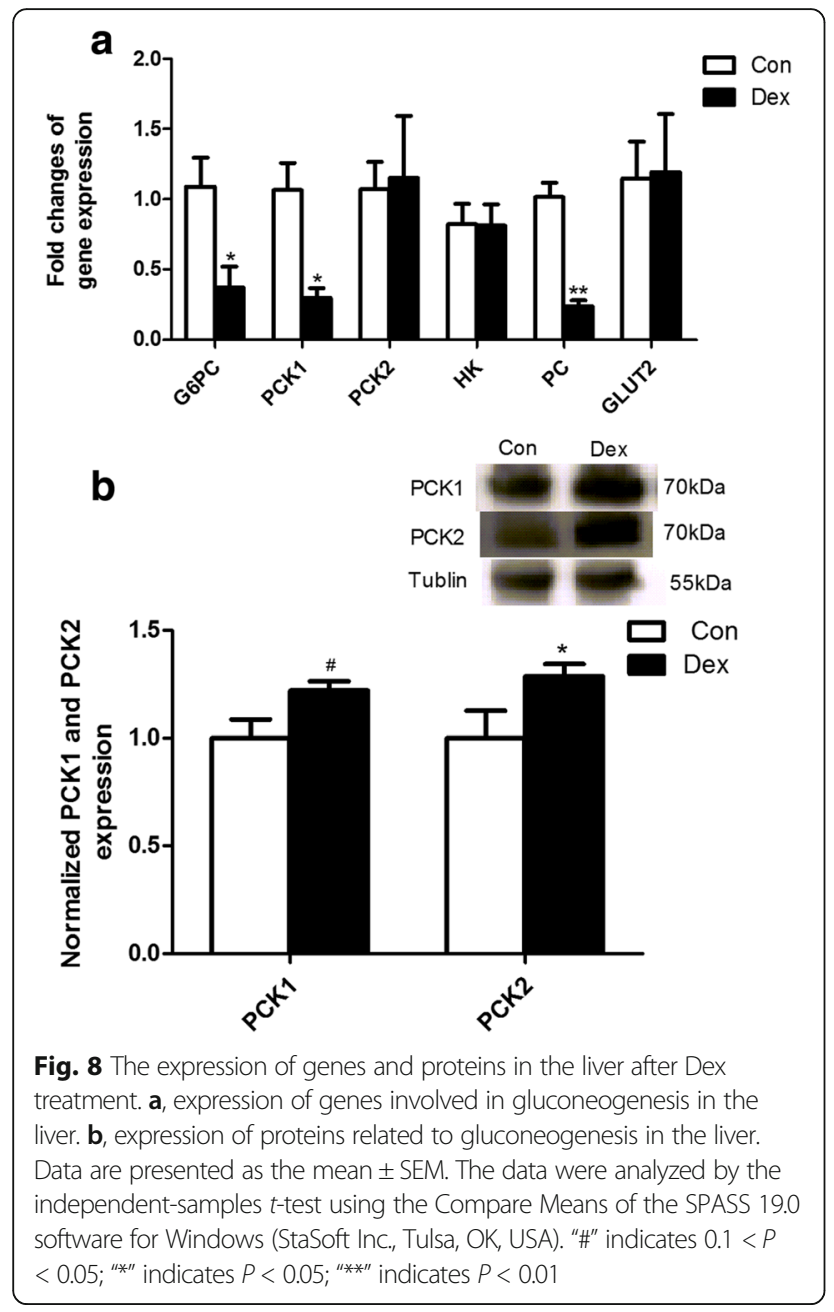

\section{Conclusions}

Our data collectively show that chronic Dex-treatment resulted in lower body weight, reduced hyperglycemia and higher hepatic glycogen accumulation in goats. According to the changes observed in hepatic genes expression, it is reasonable to assume that hyperglycemia caused by chronic exposure to Dex was mainly due to the activation of hepatic gluconeogenesis and insulin resistance.

\section{Abbreviations}

AKT: AKT serine/threonine kinase 1; Atrogin1: F-box protein 32; G6PC: Glucose-6phosphatase; GAPDH: Glyceraldehyde-3-phosphate dehydrogenase; GLUT1: Glucose transporter type 1; GLUT2: Glucose transporter type 2; GLUT4: Glucose transporter type 4; HK: Hexokinase; IGF1R: Insulin-like growth factor 1 receptor; IR: Insulin receptor; IRS1: Insulin receptor substrate1; MuRF1: Muscle RING-Finger protein 1; Na-KVATPase: Sodium-potassium ATPase; PC: Pyruvate carboxylase; PCK1: Cytosolic form of phosphoenolpyruvate carboxykinase; PCK2: Mitochondrial form of phosphoenolpyruvate carboxykinase; PI3K: Phosphoinositide 3-kinase; SGLT1: Sodium glucose transporter type 1

\section{Acknowledgements}

The authors thank the National Nature Science Foundation of China (project no. 31572433), the Program for New Century Excellent Talents in University (NCET-13-0862) and the Priority Academic Program Development of Jiangsu Higher Education Institutions (PAPD).

\section{Funding}

This work was supported by the National Nature Science Foundation of China (project no. 31572433), the Program for New Century Excellent Talents in University (NCET-13-0862) and a project funded by the Priority Academic Program Development of Jiangsu Higher Education Institutions (PAPD).

\section{Availability of data and materials}

Not applicable.

\section{Authors' contributions}

LN performed experiments and drafted the manuscript. QC CH and YG: performed experiments and analyzed the data. RZ: contributed to the experimental design and manuscript revision. LC ST and YN: conceived the idea, designed the experiment, and finalized the manuscript. All authors read and approved the final manuscript.

\section{Ethics approval}

The Institutional Animal Care and Use Committee (IACUC) of Nanjing Agricultural University approved all animal procedures. The "Guidelines on Ethical Treatment of Experimental Animals" (2006) No. 398 set by the Ministry of Science and Technology, China and the Regulation regarding the "Management and Treatment of Experimental Animals"(2008) No. 45 set by the Jiangsu Provincial People's Government, was strictly followed during the slaughtering and sampling procedures.

\section{Consent for publication}

Not applicable.

\section{Competing interests}

The authors declare that they have no competing interests.

Received: 28 August 2017 Accepted: 1 February 2018

Published online: 16 March 2018

\section{References}

1. Coplan JD, Gopinath S, Abdallah CG, Margolis J, Chen W, Scharf BA, et al. Effects of acute confinement stress-induced hypothalamic-pituitary adrenal axis activation and concomitant peripheral and central transforming growth factor-beta1 measures in nonhuman primates. Chronic Stress (Thousand Oaks). 2017;1-8

2. Tsigos C, Chrousos GP. Hypothalamic-pituitary-adrenal axis, neuroendocrine factors and stress. J Psychosom Res. 2002;53(4):865-71.

3. Shang F, Liu M, Li B, Zhang X, Sheng Y, LiU S, et al. The anti-angiogenic effect of dexamethasone in a murine hepatocellular carcinoma model by augmentation of gluconeogenesis pathway in malignant cells. Cancer Chemother Pharmacol. 2016;77(5):1087-96.

4. Poleti MD, DeRijk RH, Rosa AF, Moncau CT, Oliveira PS, Coutinho LL, et al. Genetic variants in glucocorticoid and mineralocorticoid receptors are associated with concentrations of plasma cortisol, muscle glycogen content, and meat quality traits in male Nellore cattle. Domest Anim Endocrinol. 2015;51:105-13.

5. Han W, Li J, Tang H, Sun L. Treatment of obese asthma in a mouse model by simvastatin is associated with improving dyslipidemia and decreasing leptin level. Biochem Biophys Res Commun. 2017;484(2):396-402.

6. Hasselgren P-O. Glucocorticoids and muscle catabolism. Curr Opin Clin Nutr Metab Care. 1999;2(3):201-5.

7. Annison EF, Linzell JL. The oxidation and utilization of glucose and acetate by the mammary gland of the goat in relation to their Over-all metabolism and milk formation. J Physiol. 1964;175:372-85.

8. Danfaer A, Tetens V, Agergaard N. Review and an experimental study on the physiological and quantitative aspects of gluconeogenesis in lactating ruminants. Comp Biochem Physiol B Biochem Mol Biol. 1995;111(2):201-10.

9. Overton TR, Drackley JK, Ottemann-Abbamonte CJ, Beaulieu AD, Emmert LS, Clark JH. Substrate utilization for hepatic gluconeogenesis is altered by increased glucose demand in ruminants. J Anim Sci. 1999;77(7):1940-51.

10. Ruzzin J, Wagman AS, Jensen J. Glucocorticoid-induced insulin resistance in skeletal muscles: defects in insulin signalling and the effects of a selective glycogen synthase kinase-3 inhibitor. Diabetologia. 2005;48(10):2119-30.

11. Nicod N, Giusti V, Besse C, Tappy L. Metabolic adaptations to dexamethasone-induced insulin resistance in healthy volunteers. Obes Res. 2003;11(5):625-31. 
12. Buren J, Liu HX, Jensen J, Eriksson JW. Dexamethasone impairs insulin signalling and glucose transport by depletion of insulin receptor substrate1, phosphatidylinositol 3-kinase and protein kinase B in primary cultured rat adipocytes. Eur J Endocrinol. 2002;146(3):419-29.

13. Wang ZL, Bennet WM, Wang RM, Ghatei MA, Bloom SR. Evidence of a paracrine role of neuropeptide- $Y$ in the regulation of insulin release from pancreatic islets of normal and dexamethasone-treated rats. Endocrinology. 1994;135(1):200-6.

14. O'Brien TD, Westermark $\mathrm{P}$, Johnson $\mathrm{KH}$. Islet amyloid polypeptide and insulin secretion from isolated perfused pancreas of fed, fasted, glucosetreated, and dexamethasone-treated rats. Diabetes. 1991;40(12):1701-6.

15. Jeong IK, Oh SH, Kim BJ, Chung JH, Min YK, Lee MS, et al. The effects of dexamethasone on insulin release and biosynthesis are dependent on the dose and duration of treatment. Diabetes Res Clin Pract. 2001;51(3):163-71.

16. Buckingham JC. Glucocorticoids: exemplars of multi-tasking. Br J Pharmacol. 2006;147(Suppl 1):258-68.

17. Ollier S, Beaudoin F, Vanacker N, Lacasse P. Effect of reducing milk production using a prolactin-release inhibitor or a glucocorticoid on metabolism and immune functions in cows subjected to acute nutritional stress. J Dairy Sci. 2016;99(12):9949-61.

18. Hartmann PE, Kronfeld DS. Mammary blood flow and glucose uptake in lactating cows given dexamethasone. J Dairy Sci. 1973;56(7):896-902.

19. Emikpe BO, Tanko PN, Onilude OM, Sabri MY. The influence of dexamethasone treatment and successive road transport stress on the occurrence of caprine pneumonia in a hot humid tropical environment. Veterinary World. 2013;6(8):497-501.

20. Sapolsky RM, Armanini MP, Packan DR, Sutton SW, Plotsky PM. Glucocorticoid feedback inhibition of adrenocorticotropic hormone secretagogue release. Relationship to corticosteroid receptor occupancy in various limbic sites. Neuroendocrinology. 1990;51(3):328-36.

21. Cole MA, Kim PJ, Kalman BA, Spencer RL. Dexamethasone suppression of corticosteroid secretion: evaluation of the site of action by receptor measures and functional studies. Psychoneuroendocrinology. 2000;25(2):151-67.

22. Franco-Colin M, Tellez-Lopez AM, Quevedo-Corona L, Racotta R. Effects of long-term high-sucrose and dexamethasone on fat depots, liver fat, and lipid fuel fluxes through the retroperitoneal adipose tissue and splanchnic area in rats. Metabolism. 2000;49(10):1289-94.

23. Schakman O, Kalista S, Barbe C, Loumaye A, Thissen JP. Glucocorticoid-induced skeletal muscle atrophy. Int J Biochem Cell Biol. 2013;45(10):2163-72.

24. Patel R, Magomedova L, Tsai R, Angers S, Orellana A, Cummins CL. Separating the anti-inflammatory and diabetogenic effects of glucocorticoids through LXRbeta antagonism. Endocrinology. 2017;158(4):1034-47.

25. Rafacho A, Goncalves-Neto LM, Santos-Silva JC, Alonso-Magdalena P, Merino B, Taboga SR, et al. Pancreatic alpha-cell dysfunction contributes to the disruption of glucose homeostasis and compensatory insulin hypersecretion in glucocorticoid-treated rats. PLoS One. 2014;9(4):e93531.

26. Kraus-Friedmann N. Hormonal regulation of hepatic gluconeogenesis. Physiol Rev. 1984;64(1):170-259.

27. Kuo T, Harris CA, Wang JC. Metabolic functions of glucocorticoid receptor in skeletal muscle. Mol Cell Endocrinol. 2013;380(1-2):79-88.

28. Aschenbach JR, Kristensen NB, Donkin SS, Hammon HM, Penner GB. Gluconeogenesis in dairy cows: the secret of making sweet milk from sour dough. IUBMB Life. 2010;62(12):869-77.

29. Kuo T, McQueen A, Chen TC, Wang JC. Regulation of glucose homeostasis by Glucocorticoids. Adv Exp Med Biol. 2015;872:99-126.

30. Goncalves-Neto LM, Ferreira FB, Souza L, dos Santos C, Boschero AC, Facundo VA, et al. Disruption of glucose tolerance caused by glucocorticoid excess in rats is partially prevented, but not attenuated, by arjunolic acid. Indian J Exp Biol. 2014;52(10):972-82.

31. Mokuda O, Sakamoto $Y$, Ikeda T, Mashiba H. Sensitivity and responsiveness of glucose output to insulin in isolated perfused liver from dexamethasonetreated rats. Horm Metab Res. 1991;23(2):53-5.

32. Ferrannini E, Simonson DC, Katz LD, Reichard G Jr, Bevilacqua S, Barrett EJ, et al. The disposal of an oral glucose load in patients with non-insulindependent diabetes. Metabolism. 1988;37(1):79-85.

33. DeFronzo RA, Tripathy D. Skeletal muscle insulin resistance is the primary defect in type 2 diabetes. Diabetes Care. 2009;32(Suppl 2):157-63.

34. Exton JH, Friedmann N, Wong EH, Brineaux JP, Corbin JD, Park CR. Interaction of glucocorticoids with glucagon and epinephrine in the control of gluconeogenesis and glycogenolysis in liver and of lipolysis in adipose tissue. J Biol Chem. 1972;247(11):3579-88.
35. Burke SJ, Batdorf HM, Eder AE, Karlstad MD, Burk DH, Noland RC, et al. Oral Corticosterone administration reduces Insulitis but promotes insulin resistance and hyperglycemia in male nonobese diabetic mice. Am J Pathol. 2017:187(3):614-26.

36. Drozdowski LA, lordache C, Clandinin MT, Todd Z, Gonnet M, Wild G, et al. Maternal dexamethasone and GLP-2 have early effects on intestinal sugar transport in their suckling rat offspring. J Nutr Biochem. 2009;20(10):771-82.

37. Lee CY. Chronic restraint stress induces intestinal inflammation and alters the expression of hexose and lipid transporters. Clin Exp Pharmacol Physiol. 2013;40(6):385-91.

38. Hua C, Tian J, Tian P, Cong R, Luo Y, Geng Y, et al. Feeding a high concentration diet induces unhealthy alterations in the composition and metabolism of Ruminal microbiota and host response in a goat model. Front Microbiol. 2017;8:138

39. Bai $W L$, Dang YL, Yin RH, Jiang WQ, Wang ZY, Zhu YB, et al. Differential expression of microRNAs and their regulatory networks in skin tissue of Liaoning cashmere goat during hair follicle cycles. Anim Biotechnol. 2016;27(2):104-12.

\section{Submit your next manuscript to BioMed Central and we will help you at every step:}

- We accept pre-submission inquiries

- Our selector tool helps you to find the most relevant journal

- We provide round the clock customer support

- Convenient online submission

- Thorough peer review

- Inclusion in PubMed and all major indexing services

- Maximum visibility for your research

Submit your manuscript at www.biomedcentral.com/submit
) Biomed Central 